\title{
Pre-operative Mapping and Structured Reporting of Pelvic Endometriotic Lesions on Dynamic Ultrasound and Its Correlation on Laparoscopy Using the \#Enzian Classification
}

Vimee Bindra ( $\nabla$ vimee.bindra@gmail.com )

Apollo Hospitals

Madhavi Nori

Apollo Hospitals

Girija Shankar Mohanty

Apollo Hospitals

Nivya $K$

Apollo Hospitals

Balakrishna N

Apollo Hospitals

\section{Research Article}

Keywords: \#Enzian, laparoscopic excisional surgery, pelvic endometriosis, structured reporting

Posted Date: January 21st, 2022

DOI: https://doi.org/10.21203/rs.3.rs-1272487/v1

License: (c) (i) This work is licensed under a Creative Commons Attribution 4.0 International License. Read Full License

Version of Record: A version of this preprint was published at Archives of Gynecology and Obstetrics on March 14th, 2022. See the published version at https://doi.org/10.1007/s00404-022-06494-4. 


\section{Abstract}

Purpose: The objective of this study was to evaluate and compare the diagnostic performance of ultrasonography (USG) assessment using structured reporting with intraoperative laparoscopic assessment in deep infiltrating endometriosis (DIE) using the recent update of the \#ENZIAN classification.

Methods: This was a retrospective study conducted in Tertiary Multi-disciplinary Endometriosis Care Hospital over a period of 8 months which included 50 patients who underwent a planned laparoscopic endometriosis excisional surgery after a dedicated USG assessment using International Deep Endometriosis Analysis (IDEA) protocol and \#Enzian score (updated Enzian classification), between Feb 2021 to Sept 2021 at Apollo Hospitals, Hyderabad. The pre-operative USG findings were reported in a structured reporting format and intraoperative findings were classified using the standard \#ENZIAN classification. No prospective interventions were done. A review of pre-operative ultrasound and laparoscopic findings as per the \#Enzian.

Results: Sensitivity and the negative predictive value of ultrasound were $86 \%$ and $84.2 \%$ for peritoneal lesions, $97 \%$ and $93.3 \%$ for left ovarian lesions, $93 \%$ and $91.6 \%$ for right ovarian lesions, $91 \%$ and $84 \%$ for left tubal lesions, $90 \%$ and $86.3 \%$ for right tubal lesions, $93 \%$ and $75 \%$ for uterosacral ligaments, $93.3 \%$ and $97 \%$ for rectal lesions and sensitivity and negative predictive values were $100 \%$ for rectovaginal lesions, adenomyosis, and ureteric lesions as confirmed on laparoscopy.

Conclusion: Dynamic ultrasound assessment with a structured report based on IDEA protocol and \#ENZIAN score is accurate for mapping of pelvic endometriosis in all forms, and it correlates with laparoscopic findings, thus helps surgeon for better planning and providing a road map for surgeons. From a clinical perspective, a uniform and shared reporting system across imaging and therapeutic modalities will simplify communication, improving patient management by conservative or surgical treatments, avoiding multiple repeat surgeries, and improving quality of treatment.

\section{Introduction}

Endometriosis is a complex, heterogenous, hormone dependent chronic inflammatory disorder with hall mark of extrauterine growth of endometrial gland and stroma, mainly as implants in bilateral ovaries and the pelvic peritoneum [1]. It is a polymorphic and multifocal disease with no cure or preventive mechanism.

Endometriosis incidence is $10 \%$ among reproductive age group and causes chronic and debilitating pain exacerbated during menstruation in $50 \%$ of patients and is a cause of both primary and secondary infertility in $30-40 \%$ of female with endometriosis [2,3]. Endometriosis is diagnosed late and remains undiagnosed in a large proportion of women, which results in disease progression and negative impacts on health and wellbeing [4]. Currently, gynecologists rely heavily on laparoscopy to diagnose, which results in diagnostic delays of 6-11 years [5].

The Enzian classification was published by Scientific Endometriosis Foundation-Stiftung Endometetrioseforschung (SEF) in 2003 and 2005 [6, 7]. Its revision was published in 2011. Several studies recognized it as a valid and suitable tool for mapping endometriosis lesions in ultrasonography (USG)/ magnetic resonance imaging (MRI) and surgical settings [8-10]. The new update of Enzian, also called 
\#Enzian 2021 created through a consensus process, is a comprehensive visual documentation system for a complete pictorial mapping of endometriosis including peritoneal endometriotic implants and extent of organ involvement by DIE, and is useful with both non-invasive diagnostic (USG/MRI) and laparoscopic methods, it also includes peritoneal, ovarian, and tubal lesions and extent of adnexal adhesions (Figure 1), thus providing a common language for better interpretation.

This study evaluated the diagnostic performance and reproducibility of the recent \#Enzian Score on 50 patients with deep pelvic endometriosis who underwent laparoscopic endometriosis excisional surgery.

\section{Materials And Methods}

This study was a retrospective analytical cohort study of fifty (50) patients with endometriosis who underwent laparoscopic excisional surgery for endometriosis between February 2021 to September 2021 by a single surgeon in a multidisciplinary referral hospital with vast experience in the management of endometriosis (Apollo Hospitals, Hyderabad). Preoperative ultrasound mapping was performed by a single radiologist with 15 years' experience in women's imaging using International Deep Endometriosis Analysis (IDEA) protocol and scoring the lesions as per \#Enzian in a structured reporting format [11]. Institutional review board approval was obtained for this study.

Inclusion criteria - Patients with endometriosis who underwent laparoscopic excision surgery within one month of preoperative mapping using complete ultrasound study according to the protocol detailed. Laparoscopic findings in all were classified and documented as per \#Enzian classification.

Exclusion criteria - Patients were excluded if the ultrasound was not done following the IDEA protocol and \#Enzian score by a trained sonologist or if incomplete information was available or MRI was advised before laparoscopy.

The following parameters were collected for all the patients by accessing the online medical records, and it included 1) Clinical criteria: Patient demographics including age, parity, last menstrual period, previous surgeries for endometriosis, clinical symptoms such as dysmenorrhea, dyspareunia, dysuria, dyschezia, infertility, other associated pathologies such as fibroids and polycystic ovaries, 2)Complete ultrasound assessment and mapping using predefined study protocol [Table 1] and the findings were summarized in a standard fashion using \#Enzian classification (Figure 1), 3)Laparoscopic intraoperative assessment following \#Enzian classification, documentation and histopathologic confirmation. 
Table 1

Dedicated Ultrasound protocol for evaluating pelvic endometriosis adapted from IDEA protocol (11) Dedicated Ultrasound protocol for evaluating pelvic endometriosis adapted from IDEA protocol.

1. Before commencing the ultrasound, a good clinical assessment form with mapping of specific pain sites to guide the examination to pain-specific sites for a complete thorough ultrasound assessment.

Step 1. Standard ultrasound transabdominal/transvaginal examination. This included looking at the (1) uterus (size, shape, orientation, and peri-uterine planes; endometrium; the myometrium; and the cervix), (2) the adnexa (bilateral ovaries and fallopian tubes), and (3) the cul de sac (for bowel evaluation posterior to the uterus) as we do it in routine.

But in addition to these, we also need to look at the bladder, vesicouterine pouch, the pouch of Douglas, the vagina and rectovaginal septum and bowel, rectum, and rectosigmoid for which targeted compartmental evaluation is described by the IDEA group evaluating anterior, middle and posterior compartments. This constitutes steps $2-4$ of evaluation.

Step 2. Evaluation of TV Scan "soft markers" (i.e., site-specific tenderness and ovarian mobility, and it was correlated with site-specific symptoms).

Step 3. Dynamic assessment of the status of posterior Pouch of Douglas and anterior vesicouterine pouch using real-time ultrasound-based "sliding sign".

Step 4 Assessment for Deep Infiltrating Endometriosis (DIE) nodules in anterior and posterior compartments.

Tenderness or symptom-guided site-specific USG was included wherever indicated.

\section{Statistical analysis:}

All data collected were described as quantitative numbers and percentages or mean as applicable.

Descriptive statistics like mean, percentages(\%), frequencies, positive predictive value (PPV), negative predictive value (NPV), sensitivity, specificity, and accuracy were calculated for all variables. A Chi-square test $\left(\mathbf{X}^{2}\right)$ was performed to study the association between the \#Enzian USG features and \#Enzian laparoscopic findings. The level of significance ( $\mathrm{p}$-value) is considered as 0.05 . For statistical analysis, we used IBM SPSS version 24.0 (SPSS Inc, Chicago IL)

For individual \#Enzian compartment, data was collected for the presence and absence of lesions on USG and its concordance with laparoscopic findings. Then, sensitivities and specificities for detecting endometriotic lesions in \#Enzian compartments P, O, T, A, B, C, FA, FU (Figure 1) by USG and on surgery were calculated in a specific compartment as per classification based on presence or absence of a lesion. We also calculated Negative predictive values (NPVs) and Positive predictive values (PPVs).

\section{Preoperative ultrasound Assessment, classification, and structured reporting:}

USG examination was done on a Samsung RS80 ultrasound system including a convex probe(2-5MHZ) for the abdominopelvic approach and an endovaginal probe (5-9 MHZ) for the transvaginal(TV) approach. A linear high-Resolution probe(5-12MHZ) was used when deemed necessary. All the patients after preliminary clinical assessment were subjected to ultrasound with prior appointment after bowel preparation. The timing of the scan was scheduled between day 5th to day 9th of the menstrual cycle. 
The dedicated ultrasound protocol followed for evaluating pelvic endometriosis is as detailed in Table 1

\section{Descriptors on USG and laparoscopy}

\section{Peritoneum (P)}

On USG, peritoneal lesions were interpreted as an ill definition of the peritoneal reflections over the pelvic organs, and all adhesions in the background were considered positive for peritoneal involvement. We interpreted the ill-defined peritoneal folds as positive peritoneal involvement on USG. On laparoscopy, the diameter of the virtual circle was calculated and was graded as P1, P2, or P3 as per \#ENZIAN (Figure 1). Peritoneal pseudocysts were detailed forside, lesion size and, morphology.

\section{Ovary (0)}

The endometriomas and ovarian surface implants $(\geq 5 \mathrm{~mm})$ were considered ovarian endometriosis. Lesion(s) compatible with endometrioma(s) and/or other lesions were classified as per International Ovarian Tumour Analysis (IOTA) group [12]. In case of multiloculated cysts sum of the maximal diameter was calculated on each side. Similarly, during laparoscopy, the maximal diameter was taken into consideration on either side.

\section{Tubo-Ovarian Condition ( $T$ )}

It was assessed by dynamic USG using sliding sign for either side separately of named structures, and restricted mobility or immobility between these structures was labeled as adhesions on USG. Tubal patency was not assessed in the standard protocol during transvaginal (TV) ultrasound but was checked during laparoscopy. Labeling of Tubo-ovarian condition was done using \#ENZIAN during TV scan and laparoscopy.

\section{Deep Endometriosis (DE)}

Sub-peritoneal infiltrating lesions were classified as Deep Endometriosis (DE), and as per \#ENZIAN classification, compartment-wise distribution was described.

Compartment A (vagina, rectovaginal space): the lesion was measured in sagittal section and maximum diameter of the lesion was obtained and depending on the size, it was described as A1, A2, or A3 during TV scan and laparoscopy.

Compartment B (Uterosacral ligament (USL), cardinal ligaments, and pelvic sidewall): In TV scan, the probe was introduced in the posterior vaginal fornix, and the mid sagittal plane was defined by the visualisation of cervix and its canal. By 20 degree horizontal movement of TV probe USL was visualized. Any DIE lesion identified was measured in its most lateral extent on either side and was labelled left and right separately. Diameters of all lesions were counted together on either side when multiple lesions were found. It was described as B1, B2, or B3 depending on the size (Figure 1) on TV scan and laparoscopy.

Compartment $\mathbf{C}$ (rectum): Lesions on the anterior wall of rectum were taken into consideration. All lesions within $16 \mathrm{~cm}$ of anal verge were assigned to Compartment C, and lesions above $16 \mathrm{~cm}$ were classified as 
\#Enzian Fl. It was labelled C1, C2, and C3 (Figure 1) depending on the maximal diameter of the lesion in the sagittal section along the axis of the rectum. For multifocal lesions all diameters were recorded together on USG and laparoscopy. Lesions at the rectum and sigmoid were recorded separately as C and FI.

Adenomyosis (FA) Morphological Uterus Sonographic Assessment (MUSA) criteria was used to assess adenomyosis on USG [13]. Focal adenomyosis of outer myometrium (FOAM) is a trans-serosa invading process, classically an outside to inside invasion, and was seen to involve anterior or posterior uterine peritoneal reflection. Uterine wall abnormality with an outside-in pattern of myometrial involvement with relative sparing of the junctional zone, which is typical of uterine adenomyosis, was classified in the FOAM category.

Deep lesions of the urinary bladder (FB) with involvement of the muscular layer: Bladder endometriosis shows nodular and infiltrative mural mass projecting into the bladder lumen with intact bladder mucosa. Lesions were hypoechoic on ultrasound with tethering of posterior bladder to uterus and obliteration of vesicouterine space.

Ureteric disease (extrinsic and/or intrinsic; FU): Ureteric disease was suspected when there were parametrial /retro cervical DIE lesions or ill definition and adhesions at the pelvic brim near the ovarian fossa with ureteral diameter $\geq 6 \mathrm{~mm}$ was interpreted as a congestion sign correlating to ureteric obstruction. Hydroureter or hydronephrosis coexistent with DIE was indicative of ureteric endometriosis.

Intestinal structures besides the rectum (FI) describes DIE involving lesions cranial to the rectosigmoid junction (above $16 \mathrm{~cm}$ from the anus-upper sigmoid, transverse colon, cecum, appendix, and small bowel).

Lesions on other locations- the abdominal wall, diaphragm, lung, and nerve were annotated as $F(. .$.$) and$ location in brackets as per \#Enzian classification.

The final coding was provided for each patient as follows

\#Enzian (s) assessment by ultrasound

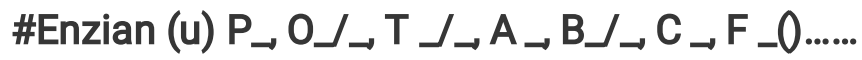

\section{Laparoscopy:}

All patients who underwent endometriosis excisional surgery were operated on by a single surgeon. The same standard technique was followed for all patients, and a \#ENZIAN score was assigned for all surgeries. All surgeries were four-port laparoscopies as per our standard practice (one $10 \mathrm{~mm}$ umbilical or supra-umbilical port and three $5 \mathrm{~mm}$ lateral ports). After a thorough inspection of the pelvis, upper abdomen, and sub-phrenic spaces, excision of endometriosis was done. The presence of endometriosis was recorded as per \#Enzian. The lesions were mapped on USG and laparoscopy as shown in Figure 2

\# Enzian (s) assessment by laparoscopy

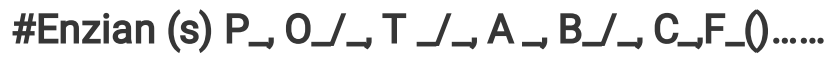

\section{Results}


A total of 50 patients who fulfilled the inclusion criteria underwent laparoscopy by a single surgeon during February 2021 to September 2021. The mean age of the patient was 32 years and patients presented with pain and infertility (Table 2). Of these 50 patients $26 \%$ (13 patients) were previously operated for endometriosis. Overall, we found that dynamic TV scan in the diagnosis of DIE, when done compartment wise is useful in most of the cases. The utility of ultrasound was found for almost all lesions but sensitivity to detect peritoneal lesions was $86 \%$ as compared to other lesions which was more than $90 \%$ for all. USG was highly accurate for rectovaginal lesions, adenomyosis and ureteric lesions. USG is also good at predicting tubal adhesions and its relationship with ovary.

Table 2

Patient Characteristics

\begin{tabular}{|ll|}
\hline Patient Characteristics & Value " $\mathrm{n}$ " (\%) \\
\hline Age, yrs mean & $32 \mathrm{yrs}$ (Range 20-42yrs) \\
\hline Parity & $36(72 \%)$ \\
\hline 0 & $14(14 \%)$ \\
\hline 1 & $12(12 \%)$ \\
\hline 2 & $2(2 \%)$ \\
\hline 3 or 4 & \\
\hline Presentation & $48(96 \%)$ \\
\hline Dysmenorrhea & $6(12 \%)$ \\
\hline Dyspareunia & $3(6 \%)$ \\
\hline Dysuria & $15(30 \%)$ \\
\hline Dyschezia & \\
\hline Infertility & $21(42 \%)$ \\
\hline Primary & $2(4 \%)$ \\
\hline Secondary & $13(26 \%)$ \\
\hline Previously operated for endometriosis & \\
\hline Values are given as $\mathrm{n}(\%)$ & \\
\hline
\end{tabular}

Preoperative US showed 24 P, 350 (Left), 260 (right), 31 T (Left), $28 \mathrm{~T}$ (right), 50 A, 38 B, 15 C, 15 FA, and 2 FU lesions ( $n=264$ lesions). Laparoscopic findings were scored with 28 P, 360 (Left), 280 (Right), $32 \mathrm{~T}$ (Left), 29 T (Right), 41 A, 40 B, 15 C, 16 FA, 2 FU lesions ( $n=267$ lesions). The overall comparison of all components and additional compartments FA, FU is presented in Table 3 
Table 3

Comparison of pre-operative US findings with laparoscopic surgical findings as reference by means of \#Enzian score

\begin{tabular}{|c|c|c|c|c|c|c|c|c|c|}
\hline LAP & $\begin{array}{l}\text { USG } \\
+\end{array}$ & USG- & $\begin{array}{l}\text { SENSTIVITY } \\
\%\end{array}$ & SPECIFICITY\% & $\begin{array}{l}\text { ACCURACY } \\
\%\end{array}$ & PPV\% & NPV\% & $x^{2}$ & $\begin{array}{l}\text { P } \\
\text { VALUE }\end{array}$ \\
\hline $\mathrm{P}+$ & 24 & 4 & 86 & 100 & 92 & 100 & 84.2 & 36.2 & .001 \\
\hline P- & 0 & 22 & & & & & & & \\
\hline LO+ & 35 & 1 & 97 & 100 & 98 & 100 & 93.3 & 45.3 & .001 \\
\hline LO- & 0 & 14 & & & & & & & \\
\hline $\mathrm{RO}+$ & 26 & 2 & 93 & 100 & 96 & 100 & 91.6 & 42.5 & .001 \\
\hline RO- & 0 & 22 & & & & & & & \\
\hline LT+ & 29 & 3 & 91 & 89 & 90 & 93 & 84 & 30.9 & .001 \\
\hline LT- & 2 & 16 & & & & & & & \\
\hline $\mathrm{RT}+$ & 26 & 3 & 90 & 90 & 90 & 92.8 & 86.3 & 31.7 & .001 \\
\hline RT- & 2 & 19 & & & & & & & \\
\hline$A+$ & 41 & 0 & 100 & 100 & 100 & 100 & 100 & 50 & .001 \\
\hline A- & 9 & 0 & & & & & & & \\
\hline$B+$ & 37 & 3 & 93 & 90 & 92 & 97.3 & 75 & 29.8 & .001 \\
\hline B- & 1 & 9 & & & & & & & \\
\hline $\mathrm{C}+$ & 14 & 1 & 93.3 & 97 & 96 & 93.3 & 97 & 40 & .001 \\
\hline C- & 1 & 34 & & & & & & & \\
\hline FA+ & 16 & 0 & 100 & 100 & 100 & 100 & 100 & 45.6 & .001 \\
\hline FA- & 1 & 33 & & & & & & & \\
\hline FU+ & 2 & 0 & 100 & 100 & 100 & 100 & 100 & 50 & .001 \\
\hline FU- & 0 & 48 & & & & & & & \\
\hline \multicolumn{10}{|c|}{ PPV $=$ Positive Predictive Value } \\
\hline \multicolumn{10}{|c|}{ NPV $=$ Negative Predictive Value } \\
\hline$P$-val & $<.05$ & s co & lered signifi & & & & & & \\
\hline
\end{tabular}

Sensitivity and negative predictive value of ultrasound confirmed by laparoscopic surgery were $86 \%$ and $84.2 \%$ for peritoneal lesions, $97 \%$ and $93.3 \%$ for left ovarian lesions, $93 \%$ and $91.6 \%$ for right ovarian lesions, $91 \%$ and $84 \%$ for left tubal lesions, $90 \%$ and $86.3 \%$ for right tubal lesions, $93 \%$ and $75 \%$ for uterosacral 
ligaments, $93.3 \%$ and $97 \%$ for rectal lesions and sensitivity and negative predictive values were $100 \%$ for rectovaginal lesions, adenomyosis, and ureteric lesions.

The $p$-value of each compartment or anatomic location mentioned was $<0.001$, and it was found to be statistically significant.

\section{Discussion}

This study was a single center retrospective study where an unbiased comparison of USG findings and intraoperative findings were made as surgeon and radiologist are different, but all cases were done by a single operator. All the patients who were confirmed with a diagnosis of DIE by laparoscopy were also diagnosed by imaging. None of the patients were diagnosed additionally on laparoscopy.

As per our study, association of other forms of endometriosis, DIE is commonly associated with ovarian and peritoneal endometriosis.

USG shows $100 \%$ specificity for 6 out of 10 parameters and more than $90 \%$ specificity for nine parameters of \#ENZIAN classification. Only for left tubal pathology, its specificity is $89 \%$. Ultrasonographic sensitivity is $100 \%$ for three parameters of \#ENZIAN classification and more than $90 \%$ for six parameters. The sensitivity of ultrasonography is $86 \%$ for peritoneal disease as compared to laparoscopic findings. For peritoneal lesions the lesions were described as an ill definition of the peritoneal reflections over the pelvic organs, and all adhesions in the background were considered positive for peritoneal involvement which can be an indirect sign of peritoneal endometriosis. For posterior compartment the superficial foci overlap the deep foci which leads to overlapping findings. For this the peritoneal involvement was taken as complete.

Recently the role of USG as a non-invasive instrument in endometriosis has shown promising results [14]. The present work demonstrates high concordance between TV scan and laparoscopic findings as per \#Enzian classification. To our knowledge, this is the first of its kind of work to find a correlation between USG findings and laparoscopic findings as per the recent update of Enzian, also called \#Enzian, after its first publication in January 2021 [9]. Our study found that DIE detected by USG and laparoscopic \#Enzian score were similar, making ultrasonography a feasible diagnostic tool to plan for adequate excision of endometriosis. Moreover, ultrasound findings can suggest the best surgical strategy for retro-pelvic endometriosis and other DIE.

The chronic nature of the disease mandates a lifelong personalized treatment plan with a goal of optimizing the use of limited medical therapies available for endometriosis and curtailing repeated surgical procedures. The treatment decisions are complicated and are influenced by patient characteristics, the disease location and extent as determined by USG or imaging, medication cost / side effects, and surgical cost /need for surgery. However, it is evident at each level of treatment decision and planning, accurate diagnostic assessment is crucial as it involves multi-disciplinary team involvement for complete surgical excision.

There is a gap in the standard of endometriosis care, which demands comprehensive and structured reporting of USG in routine clinical practice [15]. The use of a structured reporting system and common language of communication between sonologist and surgeon will enable both to assess every compartment in detail, and in turn, this will avoid diagnostic delays in endometriosis care and to plan surgical time and approach as well. 
Sufficient accuracy of dedicated meticulous protocol on TV scan provides good diagnostic performance in identification and mapping across the severity and spectrum of disease and is proven as a replacement test for deep pelvic endometriosis diagnosis rendering diagnostic laparoscopy obsolete [16]. The International Society of Gynecologic Endoscopy (ISGE) structured reporting template is an amalgamation of highly differentiated evaluation based on IDEA protocol and structured documentation of the USG findings based on the \#Enzian classification which can be used by the surgeon [15]. This merging of advances and innovations in the field of ultrasound diagnostics and laparoscopy results in significantly simplified communication and improving both management and quality control for conservative and surgical treatments. It also simplifies patient counselling, treatment planning, discussion regarding complications, inclusion of multidisciplinary teams for bowel, ureter, bladder and extra-pelvic sites and opting for the most suitable surgical therapeutic strategy.

Limitations of this study are its retrospective study design and small sample population. further prospective studies with structured USG reporting and consistent protocols are needed in a multi-centric setting. The peritoneal involvement needs more dedicated studies in future for determining the diagnostic accuracy of USG for superficial lesions as there is a significant overlap of superficial and deep peritoneal endometriosis in posterior compartment.

Dynamic ultrasound assessment with a structured report based on IDEA protocol and \#ENZIAN score is accurate for topographic mapping of pelvic endometriosis in all forms, and it correlates with the laparoscopic findings, thus enabling better planning and providing a road map for surgeons. This is accurate, reproducible and, correlates with the laparoscopic findings with endometriosis and or DIE, adenomyosis, and pelvic adhesions. Standardization of USG protocols and reporting will be a crucial step towards early diagnosis of endometriosis and intervention, which in turn will avoid delay in endometriosis diagnosis, and \#ENZIAN Score may be used as an effective communication tool between gynecologists and radiologists for the standard of care which in turn will promote treatment effectiveness, patient safety and will lower the chances of incomplete excision and repeat surgeries.

\section{Declarations}

\section{Acknowledgements:}

We would like to thank Prof Jorg Keckstein for his guidance and inputs in this study and granting permission to reproduce \#EnzianClassification

\section{Disclosures:}

NONE

\section{Author Contribution:}

All authors contributed to the study conception and design. Material preparation, data collection were performed by Vimee Bindra, Nori Madhavi, Girija Shankar Mohanty, Nivya K. Statitical analysis and interpretation of results was performed by Balakrishna N. The first draft of the manuscript was written by 
Vimee Bindra and all authors commented on previous versions of the manuscript. All authors read and approved the final manuscript.

\section{Conflict of interest:}

The authors declare no conflict of interest.

\section{References}

1. Corrêa TL, Lins ID, Moura MJC, López Droguett E. Generalized renewal process based on the q-weibull distribution for reliability applications. Risk, Reliab Saf Innov Theory Pract - Proc 26th Eur Saf Reliab Conf ESREL 2016. 2017;362(25):175. spondence should be addressed.Entropy 2018, 20(4), 223; https://doi.org/10.3390/e20040223

2. Triolo. Chronic Pelvic Pain in Endometriosis: An Overview. J Clin Med Res. 2013;5(3):153-63. https://doi.org/10.4021/jocmr1288w

3. Rogers PAW, D’Hooghe TM, Fazleabas A, Giudice LC, Montgomery GW, Petraglia F, et al. Defining future directions for endometriosis research: Workshop report from the 2011 World Congress of Endometriosis in Montpellier, France. Reprod Sci. 2013;20(5):483-99. doi: https://doi.org/10.1177/1933719113477495

4. Agarwal SK, Chapron C, Giudice LC, Laufer MR, Leyland N, Missmer SA, et al. Clinical diagnosis of endometriosis: a call to action. Am J Obstet Gynecol [Internet]. 2019;220(4):354.e1-354.e12. Available from: https://doi.org/10.1016/j.ajog.2018.12.039

5. Nnoaham KE, Hummelshoj L, Webster P, d'Hooghe T, de Cicco Nardone F, de Cicco Nardone C, et al. Reprint of: Impact of endometriosis on quality of life and work productivity: a multicenter study across ten countries. Fertil Steril. 2019;112(4):e137-52. https://doi.org/10.1016/j.fertnstert.2011.05.090.

6. Tuttlies F, Keckstein J, Ulrich U, Possover M, Schweppe KW, Wustlich M, et al. ENZIAN-Score, eine klassifikation der tief infiltrierenden endometriose. Zentralbl Gynakol. 2005;127(5):275-81. https://doi.org/10.1055/s-2005-836904.

7. Keckstein J, Hudelist G. Classification of deep endometriosis (DE) including bowel endometriosis: From rASRM to \#Enzian-classification. Best Pract Res Clin Obstet Gynaecol. 2021;71:27-37. https://doi.org/10.1016/j.bpobgyn.2020.11.004

8. Hudelist G, English J, Thomas AE, Tinelli A, Singer CF, Keckstein J. Diagnostic accuracy of transvaginal ultrasound for non-invasive diagnosis of bowel endometriosis: Systematic review and meta-analysis. Ultrasound Obstet Gynecol. 2011;37(3):257-63. https://doi.org/10.1002/uog.8858.

9. Keckstein J, Saridogan E, Ulrich UA, Sillem M, Oppelt P, Schweppe KW, et al. The \#Enzian classification: A comprehensive non-invasive and surgical description system for endometriosis. Acta Obstet Gynecol Scand. 2021;100(7):1165-75. https://doi.org/10.1111/aogs.14099.

10. Hudelist G, Montanari E, Salama M, Dauser B, Nemeth Z, Keckstein J. Comparison between Sonographybased and Surgical Extent of Deep Endometriosis Using the Enzian Classification - A Prospective Diagnostic Accuracy Study. J Minim Invasive Gynecol [Internet]. 2021;28(9):1643-1649.e1. Available from: https://doi.org/10.1016/j.jmig.2021.02.009 
11. Guerriero S, Condous G, van den Bosch T, Valentin L, Leone FPG, Van Schoubroeck D, et al. Systematic approach to sonographic evaluation of the pelvis in women with suspected endometriosis, including terms, definitions and measurements: a consensus opinion from the International Deep Endometriosis Analysis (IDEA) group. Ultrasound Obstet Gynecol. 2016;48(3):318-32.

https://doi.org/10.1002/uog.15955

12. Timmerman D, Valentin L, Bourne TH, Collins WP, Verrelst $H$, Vergote I. Terms, definitions and measurements to describe the sonographic features of adnexal tumors: A consensus opinion from the International Ovarian Tumor Analysis (IOTA) group. Ultrasound Obstet Gynecol. 2000;16(5):500-5. https://doi.org/10.1046/j.1469-0705.2000.00287.x

13. Van Den Bosch T, Dueholm M, Leone FPG, Valentin L, Rasmussen CK, Votino A, et al. Terms, definitions and measurements to describe sonographic features of myometrium and uterine masses: A consensus opinion from the Morphological Uterus Sonographic Assessment (MUSA) group. Ultrasound Obstet Gynecol. 2015;46(3):284-98. https://doi.org/ 10.1002/uog.14806

14. Pattanasri M, Ades A, Nanayakkara P. Correlation between ultrasound findings and laparoscopy in prediction of deep infiltrating endometriosis (DIE). Aust New Zeal J Obstet Gynaecol. 2020;60(6):946-51. https://doi.org/ 10.1111/ajo.13242

15. Djokovic D, Pinto P, van Herendael BJ, Laganà AS, Thomas V, Keckstein J. Structured report for dynamic ultrasonography in patients with suspected or known endometriosis: Recommendations of the International Society for Gynecologic Endoscopy (ISGE). Eur J Obstet Gynecol Reprod Biol [Internet]. 2021;263:252-60. Available from: https://doi.org/10.1016/j.ejogrb.2021.06.035

16. Holland TK, Cutner A, Saridogan E, Mavrelos D, Pateman K, Jurkovic D. Ultrasound mapping of pelvic endometriosis: Does the location and number of lesions affect the diagnostic accuracy? A multicentre diagnostic accuracy study. BMC Womens Health [Internet]. 2013;13(1):1. Available from: BMC Women's Health https://doi.org/ 10.1186/1472-6874-13-43

\section{Figures}




\section{\# Enzian}

\section{(Classification of Endometriosis)}
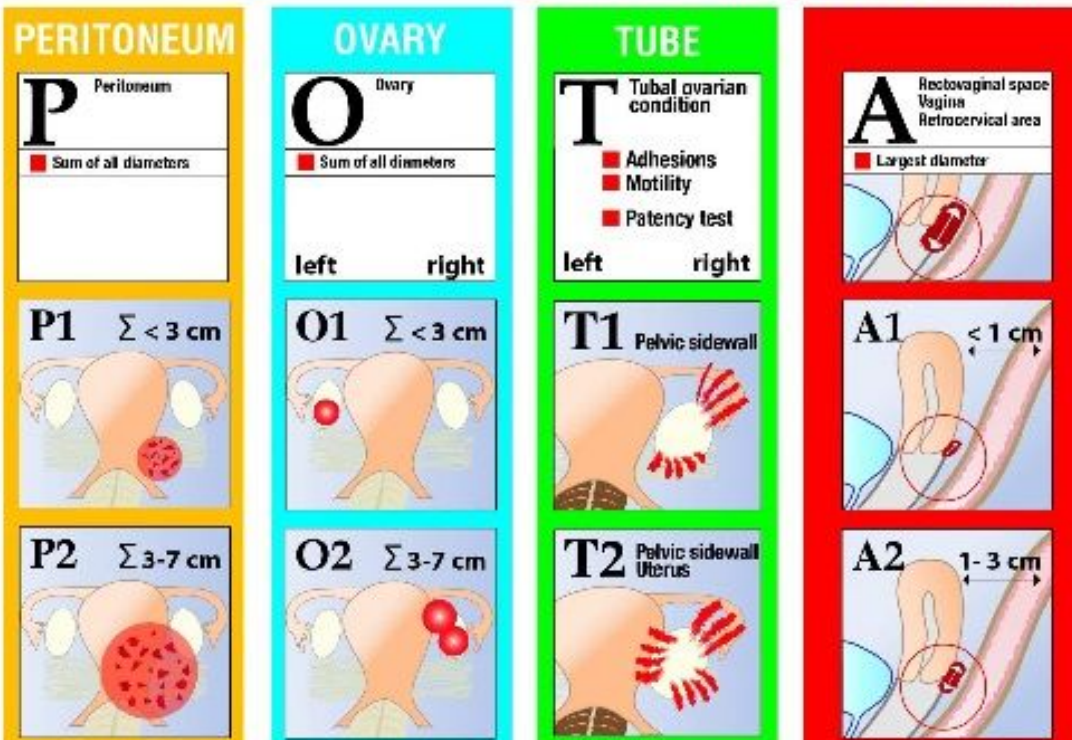

DEEP ENDOMETRIOSIS

$\mathrm{O} 2 \sum \sum_{3-7} \mathrm{~cm}$
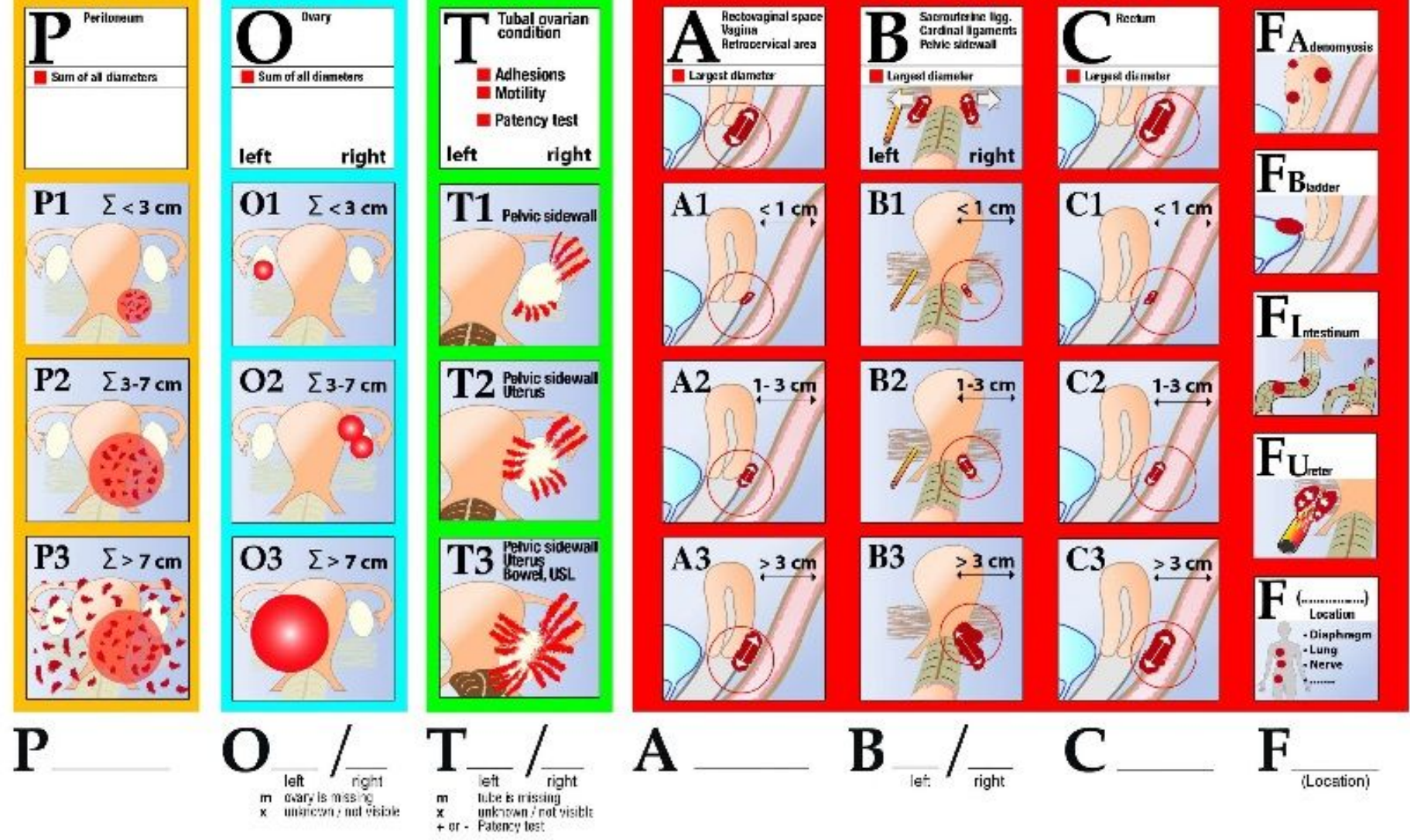

A

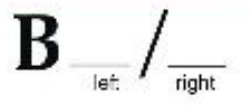

C

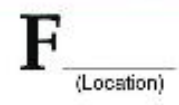

\section{Figure 1}

\#Enzian system - an overview with potentially affected organs and compartments (reproduced with permission from Keckstein et al., 2021 (9)). 

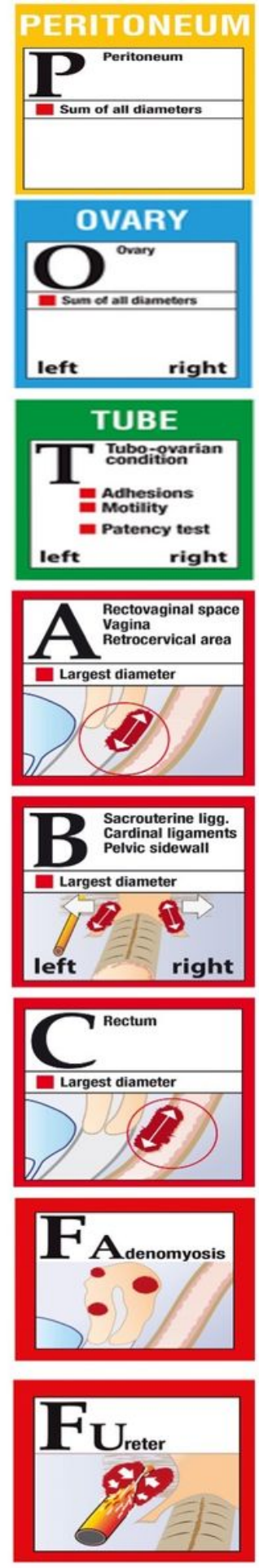
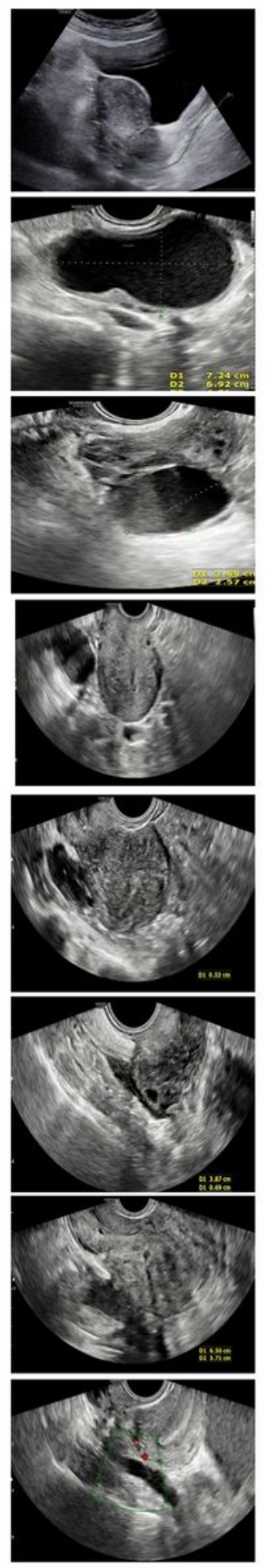
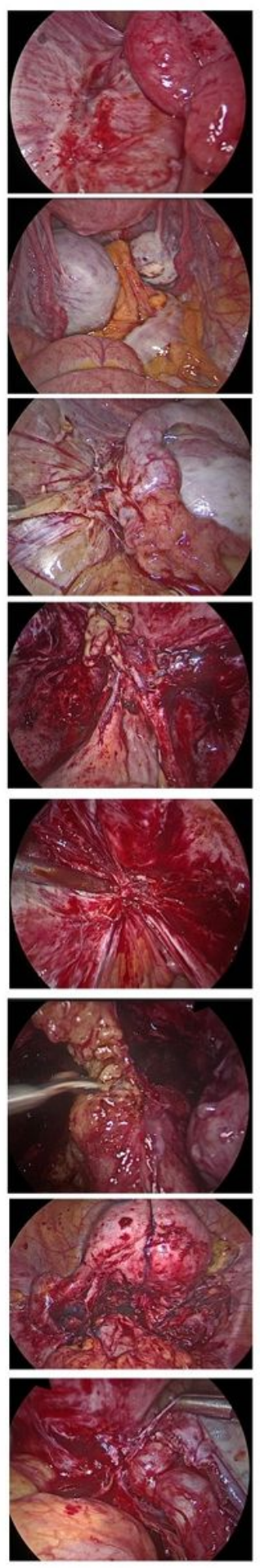

Figure 2

Compartment wise mapping of lesions on dynamic ultrasound and laparoscopy following \#Enzian classification 